necessary to distinguish change of absorption of the aromatic amino-acids from appearance of new absorbing centres (CO groups) before such observations could be used as arguments for or against the cyclol hypothesis.

The question will be discussed more fully in a paper to be published shortly.

Research Laboratories,

Ensor Holmday.

London Hospital, E.1.

'Haurowitz and Astrup, NatUre, 143, 119 (1939).

\section{Protein Stability as Revealed by Ultracentrifugal Analysis}

In the course of sedimentation velocity studies with serum protein mixtures, it has been noted from time to time that the concentrations of the different molecules calculated from the sedimentation diagram differ from those determined analytically ${ }^{1}$. The substance sedimenting at the higher rate decreases in amount to form units of a lower apparent sedimentation constant, even though it has appeared to be perfectly stable under the same conditions when it is present alone. It has been concluded that these changes are caused by some medium effect produced by the action of one protein upon the other. It was then reasoned that it should be possible to produce this effect by means of such lower molecular substances as amides, amino-acids, polypeptides, and protamines. The action of such 'dissociating compounds' is found to be highly specific. Thus an amino-acid which acts strongly upon a certain protein may have no effect upon another, and vice versa, and the effect may depend upon the presence or absence of salt ${ }^{2,3}$.

Naturally it is important to understand such effects of proteins upon each other and of smaller molecules upon proteins. In some quarters it is believed the effect is due to a real dissociation of the faster sedimenting unit, but it must be recognized that in some cases the modification of the sedimentation rate may arise from a change in the shape of the molecule or even from hydration effects. It

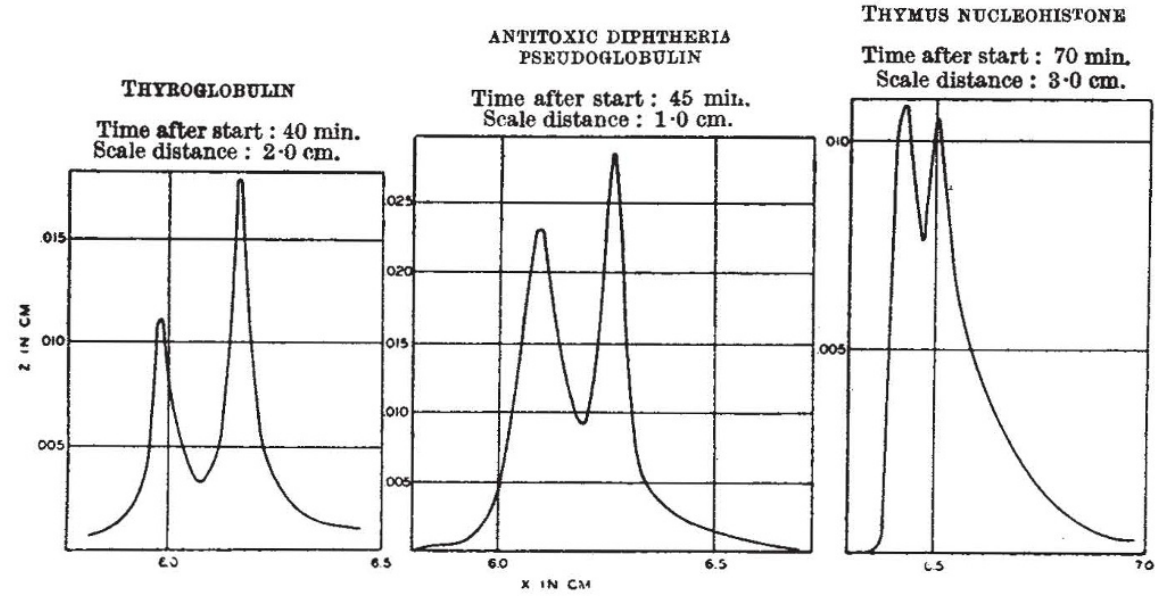

ULTRACENTRIFUGAL SEDIMENTATION DIAGRAMS.

From left to right in each diagram, labile and native components. Concentration and $p \mathrm{H}$ for each protein as follows: thyroglobulin, 4.3\%,pH 6.1; antitoxic diphtheria pseudoglobulin, $5 \cdot 5 \%, p \mathrm{H} .7 \cdot 6$; thymus nucleohistone, $1 \cdot 2 \%, p \mathrm{H} 6 \cdot 0$. appears that final answer to this question will have to come from the results of parallel osmotic pressure determinations.

It has now been found that changes in sedimenta. tion behaviour of the type mentioned above can be produced, at least in solutions of the uniform proteins now being investigated in this Laboratory (antitoxic (diphtheria) pseudoglobulin, thyroglobulin', thymus nucleohistone), by the simple expedient of increasing the protein concentration in the solution and reducing the electrolyte content. In other words, the effect is produced without the addition of any reagent. Typical sedimentation diagrams are shown in the accompanying figure. There are many reasons for believing this is a quite general property of protein systems.

The observations can be described by means of the following scheme :

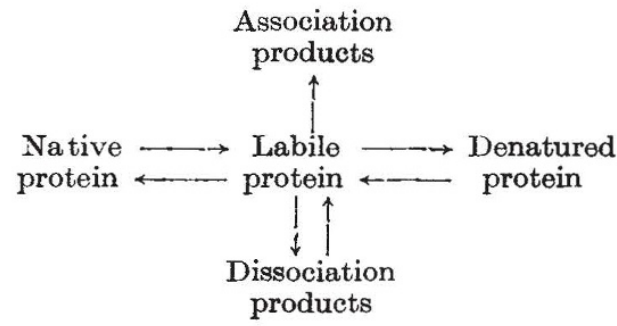

The formation of the labile state, probably due to an unfolding of native protein, appears not to involve any reaction between molecules, but is essentially a slow, intramolecular change involving an equilibrium, the rate of establishment of which depends upon protein and hydrogen ion concentration of the solution and temperature. An increase in protein concentration and the removal of electrolyte from the solution favour the formation of the labile form. Any subsequent formation of denatured protein, dissociation or association products depends upon the presence of such reagents as urea, salicylate, hydrogen ion concentration of the solution, heat, ultra-violet light, and other factors.

Inasmuch as the presence of certain proteins, amino-acids or other substances favours the establishment of this equilibrium or may cause complete denaturation or change in molecular size even in the presence of salt, we believe this equilibrium between native and labile protein to form the basis of effects noticed by McFarlane, Pedersen, and others.

HAROLD P. LUNDGREN. Laboratory of

Physical Chemistry, University of Wisconsin, Madison.

April 4.

'McFarlane, Biochem. J., 29, 407 (1935).

3 Pedersen, Nature, 138, 363 (1936).

s Svedberg, Ind. Eng. Chem., Anal. Ed., 10, 119 (1938).

4 Lundgren, J. Chem. Phys., 6, 177 (1938). 